Article

\title{
New Insight into the Mechanism of Drug Release from Poly(D,L-lactide) Film by Electron Paramagnetic Resonance
}

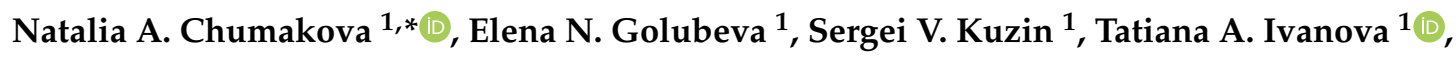 \\ Igor A. Grigoriev ${ }^{2}$, Sergey V. Kostjuk ${ }^{3,4,5}{ }^{\mathbb{D}}$ and Mikhail Ya. Melnikov ${ }^{1}$ \\ 1 Department of Chemistry, M. V. Lomonosov Moscow State University, Leninskiye Gory, 1/3, \\ 119991 Moscow, Russia; legol@mail.ru (E.N.G.); ser.12.08@yandex.ru (S.V.K.); \\ tatianaivanovamsu@gmail.com (T.A.I.); melnikov46@mail.ru (M.Y.M.) \\ 2 Vorozhtsov Novosibirsk Institute of Organic Chemistry Siberian Branch, Russian Academy of Sciences, \\ Lavrentiev Ave., 9, 630090 Novosibirsk, Russia; grig@nioch.nsc.ru \\ 3 Department of Chemistry, Belarusian State University, Leningradskaya Str. 14, 220006 Minsk, Belarus; \\ kostjuks@bsu.by \\ 4 Research Institute for Physical Chemical Problems, Belarusian State University, 14 Leningradskaya Str., \\ 220006 Minsk, Belarus \\ 5 Institute for Regenerative Medicine, Sechenov First Moscow State Medical University, 119991 Moscow, Russia \\ * Correspondence: harmonic2011@yandex.ru; Tel.: +7-916-797-4879
}

Received: 11 November 2020; Accepted: 14 December 2020; Published: 18 December 2020

\begin{abstract}
A novel approach based on convolution of the electron paramagnetic resonance (EPR) spectra was used for quantitative study of the release kinetics of paramagnetic dopants from poly(D,L-lactide) films. A non-monotonic dependence of the release rate on time was reliably recorded. The release regularities were compared with the dynamics of polymer structure changes determined by EPR, SEM, and optic microscopy. The data obtained allow for the conclusion that the main factor governing dopant release is the formation of pores connected with the surface. In contrast, the contribution of the dopant diffusion through the polymer matrix is negligible. The dopant release can be divided into two phases: release through surface pores, which are partially closed with time, and release through pores initially formed inside the polymer matrix due to autocatalytic hydrolysis of the polymer and gradually connected to the surface of the sample. For some time, these processes co-occur. The mathematical model of the release kinetics based on pore formation is presented, describing the kinetics of release of various dopants from the polymer films of different thicknesses.
\end{abstract}

Keywords: poly(D,L-lactide) films; paramagnetic dopants; electron paramagnetic resonance; mechanism of drug release; mathematical model of the release kinetics

\section{Introduction}

Biodegradable polymers are the class of materials widely used in medicine that degrade in a body environment without producing toxic compounds [1]. An application area of a particular polymer is determined mainly by the rate of its dissolution and/or degradation in vivo. Polymer materials that decompose within a few hours (e.g., hydroxyethylcellulose) are used for the short-term delivery of medical substances [2]. Polymer forms that are stable in the body for several weeks or months (for example, polylactide and poly(lactide-co-glycolide) copolymers) are applied for the production of temporary prostheses [3,4] as well as for the creation of long-acting drug delivery systems [5].

Biodegradable polymers for medical purposes can be loaded with various bioactives-growth factors, anti-inflammatory drugs, etc. [6-11]. In this paper, such additives are denoted as dopants. One of 
the most promising approaches for creating doped polymer materials is supercritical fluid technologies. The property of polymers to swell or dissolve in supercritical solvents is used to encapsulate various bioactives. In a ternary system, "supercritical fluid-polymer-dopant", a transition of the dopant molecules from the supercritical fluid solution into the swollen polymer occurs. Upon depressurization, the solvent gaseous at ambient conditions leaves the polymer matrix ultimately. Such a technique allows for obtaining the doped polymer free of toxic solvent traces. At present, supercritical $\mathrm{CO}_{2}$ $\left(\mathrm{scCO}_{2}\right)$ is the most common supercritical solvent combining mild critical parameters $\left(\mathrm{P}_{\mathrm{c}}=74\right.$ bar and $\mathrm{T}_{\mathrm{c}}=307 \mathrm{~K}$ ) with low toxicity. The techniques based on $\mathrm{scCO}_{2}$ are used to encapsulate organic molecules: drugs [12], dyes [13], organometallic compounds (followed by thermal, chemical, or radiation treatment of the material to obtain metal-polymer nanocomposites [14,15]), monomers, and initiators for the production of polymer composites [16,17].

One of the essential characteristics of doped polymers for biomedical purposes is the drug release kinetics in vitro and in vivo [18-22]. Kinetic regularities depend on the nature of the polymer, the dopant, and the dosage form or prostheses' shape and structure. In the case of medical materials doped with medicinal substances, the goal is achieving linear dependence of a drug release on time, that is, the creation of materials characterized by uniform release. Release from a porous matrix is, to a great extent, determined by diffusion of the dopant molecules through the pores and depends on the pores' size, shape, and permeability. The factors influencing the release kinetics from micronized polymers are the shape, size, and ability of the particles for self-adhesion [22].

Mathematical description of the release of different substances from matrices of aliphatic polyesters is a complicated task due to many physicochemical factors to consider. The most important are modifications of the material structure during swelling and hydrolysis, changes in the diffusion coefficients of the dopant and hydrolysis products during plasticization of the polymer, complex kinetic patterns of hydrolytic degradation of the matrix, etc. Therefore, some authors use a phenomenological description of the release $[23,24]$. Therefore, for example, on the basis of experimental data analysis on the release of dopants from samples of various architectonics, the authors of [23] proposed the power law. To describe the kinetic curves of dopant release including the induction period, an additional parameter (the lag time) was included corresponding to the time of onset of the release of the substance [24-26]. Another modification of the model considers a sharp initial release (so-called "burst") [27]. This phenomenon is usually attributed to the washout of the substance from the surface of the matrix, but this is contrary to the fact that the release can last for several days [28]. More modern models take into account changes in the diffusion coefficients of the dopants due to plasticization of the polymer matrix as a result of swelling in water or due to a decrease in molecular weight during hydrolysis (see, for example, [26,29]). Models that take into account diffusion in pores formed in polymer matrices seem to be more correct $[30,31]$. However, the available physicochemical models are primarily based not only on experimental data but also on speculative assumptions not supported by experiments. In this regard, it remains relevant to obtain information on changes in the polymer matrix structure at the macro and micro levels.

Electron paramagnetic resonance (EPR) spectroscopy is a promising method for the study of the release mechanism. This method allows for observing not only the paramagnetic dopant molecules released into the external environment but also the molecules remaining inside the polymer matrix [32-34]. The use of stable nitroxide radicals as paramagnetic dopants allows for observing the mobility of dopant molecules inside the polymer at various stages of the release process. It is known that nitroxide radicals are stable in different polymeric matrices during several months [35-37]. In our previous work [35], we demonstrated the possibility of doping polylactide with nitroxide radicals using supercritical carbon dioxide.

A serious obstacle to using EPR spectroscopy to study dopant release from polyester materials is the autocatalytic hydrolysis of these polymers producing aliphatic acids [38,39]. To prevent acidification, it is necessary to use a buffer solution and to replace it regularly, which results in a low concentration of the paramagnetic compound in the external environment. Moreover, due to the high dielectric constant 
of water, the EPR probe volume should be small. When the number of spins in the probe is less than approximately $10^{13}$, the EPR spectrum has a low signal-to-noise ratio, which leads to low accuracy in determining the radical's concentration by the standard method based on double integration of the spectrum. For this reason, most works devoted to the release of spin probes from polylactide or poly(lactide-co-glycolide) matrices contain only qualitative analysis of the dynamics of changes in the EPR spectra.

In this paper, the novel approach for obtaining quantitative information from highly noisy EPR spectra was applied to study the features of the release of paramagnetic dopants-nitroxide spin probes, including spin-labeled diclofenac, from poly(D,L-lactide) films to aqueous media. In [35], we show the effectiveness of this method for investigation of the release regularities of spin probes from micronized poly(D,L-lactide). Unfortunately, modeling of the release process from micronized polymer forms is rather complicated. The best way to determine the fundamental regularities of the release kinetics determined by the nature of the polymer and the drug is to investigate the structures with easily reproduced geometry—cylindrical forms, tablets, or films. The last are the most promising since, in this case, the diffusion problem can be regarded as a one-dimensional. It is the release from polymer films that was studied in this work.

The accurately measured release curves were compared with the changes in the rotational mobility of paramagnetic molecules inside the polymer during polymer swelling and hydrolysis. The use of $\mathrm{pH}$-sensitive spin probes allowed for revealing local acidity inside the polymer. Based on the results obtained, one can conclude that the foreground of the dopant release from poly(D,L-lactide) is diffusion through the liquid filling the pores inside the polymer; the contribution of dopant diffusion through the polymer matrix is negligible. The mathematical model of the release kinetics, including two stages of pore formation, was developed.

\section{Materials and Methods}

\subsection{Materials and Substances}

End-caped poly(D,L-lactide) (PDL 04 and PDL 02) was purchased from PURASORB (Netherlands). Stable nitroxide radicals 4-hydroxy-2,2,6,6-tetramethylpiperidine-1-oxide (TEMPOL) and 4-oxo-2,2,6,6-tetramethylpiperidine-1-oxide (TEMPONE) from Sigma-Aldrich were used without further purification. The $\mathrm{pH}$-sensitive spin probes 4-amino-2,2,5,5-tetramethyl-2,5-dihydroimidazole-1-oxyl (ATI) and 5,5-dimetyl-4-dimetylamino-2-ethyl-2-(4-pyridyl)-2,5-dyhydroimidazole-1-oxyl (DPI) (purity > 96\%) were synthesized according to experimental procedures described in $[40,41]$, respectively. Spin-labeled bioactive diclofenac (sl-DCF) (purity $>95 \%$ ) was provided to us by Dr. T. Kalai (University of Pécs, Pécs, Hungary); the methodic of synthesis is given in [35]. The structures of the paramagnetic molecules are presented in Figure 1.

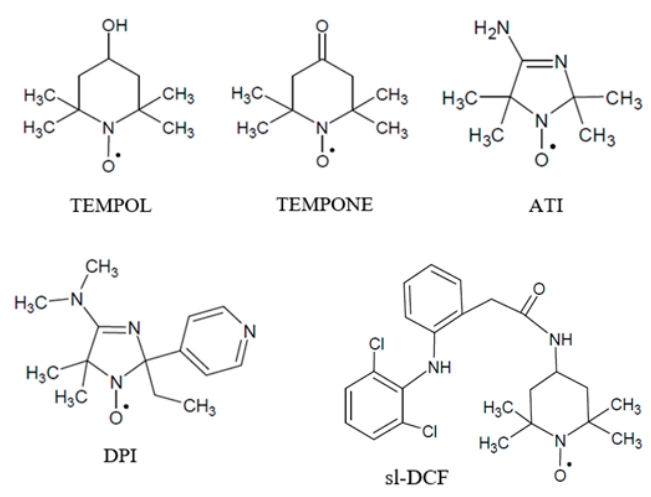

Figure 1. The structures of the paramagnetic compounds. 
Toluene (Komponent Reaktiv, Moscow, Russia) was distilled over metallic sodium. Phosphate-buffered saline (PBS) was prepared by dissolving the tablets from Pushchinskiye Laboratorii, Moscow region, Russia, in distilled water. $\mathrm{pH}$ of the prepared PBS was monitored using a pre-calibrated pH meter/millivoltmeter. Chemically pure carbon dioxide (99.998\% grade, NIIKM Ltd., Moscow, Russia) was applied without additional purification.

\subsection{Samples Preparation}

In the first step, the SCF mini-laboratory [42] was used to fabricate porous polymeric matrices of PDL 04 and PDL 02 impregnated by paramagnetic spin probes in $\mathrm{scCO}_{2}$ solution. A detailed procedure for the preparation of polymeric porous materials is described in [2]. The supercritical fluid process's optimal parameters are as follows: temperature $41-43^{\circ} \mathrm{C}$, pressure $160 \mathrm{MPa}$, holding time $8 \mathrm{~h}$, and pressure release time 50-70 min. Earlier, we showed [43] that the used method allows for forming the samples with uniform distribution of the dopant in the polymer at the macroscopic and molecular levels. The dopant concentration in the polymer was $(1-5) \times 10^{-5} \mathrm{~mol} / \mathrm{g}$ in order to reduce the dipole-dipole distortion of EPR spectra. In the case of DPI/PDL 02, the dopant concentration was $3.6 \times 10^{-6} \mathrm{~mol} / \mathrm{g}$. In the second step, the porous matrices doped with nitroxides were subjected to grinding in a knife mill, sieving through a sieve with a mesh size of $<100 \mu \mathrm{m}$ and subsequent hot $(333 \mathrm{~K})$ pressing under a pressure of $2 \mathrm{t} / \mathrm{sm}^{2}$ for approximately $30 \mathrm{~s}$ (Specac Atlas Manual Hydraulic Press equipment $(25 \mathrm{t})$ ). The particles had broad size distribution; however, the predominant fraction was particles with $40-60 \mu \mathrm{m}(48 \%)$. To create a film with a thickness of $200-240 \mu \mathrm{m}, 0.30-0.35 \mathrm{~g}$ of powder was used.

Quality control of the polymer films was carried out by optical and scanning electron microscopy. Optical microscopy experiments were performed using the Soptop CX40M metallographic microscope, NINGBO SUNNY INSTRUMENT CO., LTD., Zhejiang, China. The dry and swollen films' surfaces were visualized by the JCM-6000 Neoscope scanning electron microscope, JEOL Ltd., Tokyo, Japan, without gold sputtering. The optimal accelerating voltage not deforming the samples was found to be $10 \mathrm{kV}$. It is necessary to note that a large number of samples were analyzed and that the most typical images are presented in the article.

\subsection{Release Experiments}

Film fragments of sizes $25-40 \mathrm{~mm}^{2}$ were placed into $0.5 \mathrm{~mL}$ of PBS (pH 7.3) and kept in orbital shaker-incubator ES-20 (Biosan, Latvia, $250 \mathrm{rpm}$ ) at $310 \mathrm{~K}$. Regular (once a day or two) change of the solution was performed to avoid acidification due to hydrolytic degradation of poly $(\mathrm{D}, \mathrm{L}-\mathrm{lactide})[38,39]$. The dopant release was controlled by determining of the number of radicals in the samples (approximately $5 \mu \mathrm{L}$ ) taken from the external medium at predetermined times (see below). Due to the replacement of PBS, the release kinetics was measured in differential form. The cumulative kinetic curve was be calculated by summation of the released radical amount.

For studying processes inside the films during swelling and hydrolysis of the polymer, the samples were periodically removed from the liquid and dried with a napkin. Then, the content of paramagnetic substance was measured, and the shape of the EPR spectrum of radicals in the films was analyzed. The structure of the surface and the near-surface layer of the swollen film were studied by SEM and optical microscopy, respectively.

\subsection{EPR Spectroscopy}

\subsubsection{Spectra Recording}

EPR spectra were recorded by EPR spectrometer Bruker EMX-500 (Bruker, Karlsruhe, Germany). The thermostatic device of Bruker was used. Liquid samples were placed into glass tubes with an inner diameter of $0.9 \mathrm{~mm}$ or $1.6 \mathrm{~mm}$; the samples' height did not exceed $4 \mathrm{~mm}$. Film fragments were studied 
in quartz tubes with an inner diameter of $4.0 \mathrm{~mm}$. Spectra were recorded at microwave radiation power, not leading to signal saturation.

\subsubsection{Numerical Analysis of EPR Spectra}

The amount of paramagnetic substance in the polymer samples was defined using the standard method of EPR spectra double integration [44]. The number of radicals in liquid probes cannot be determined using this method for two reasons. Firstly, the outer solution replacement leads to a low concentration of radicals. Secondly, a high dielectric constant of water does not permit studying samples exceeding 4-5 $\mu \mathrm{L}$. As a result, the average number of radicals in the liquid probe did not exceed $10^{12}-10^{13}$; the EPR spectra of such samples have a meager signal-to-noise ratio (see Figure 2). That is why our team's method developed [45] was applied for the spectra's quantitative analysis. The approach is based on convolution of the experimental EPR spectra with the spectrum of the same system "radical-solvent" characterized by a high signal-to-noise ratio. As an example, Figure 2 demonstrates the result of convolution of the EPR spectrum of a liquid probe containing TEMPOL with the spectrum of this radical in PBS. It is seen that a much higher signal-to-noise ratio characterizes the convolution function, and that its intensity can be determined with a smaller error than one of the noisy EPR signal.

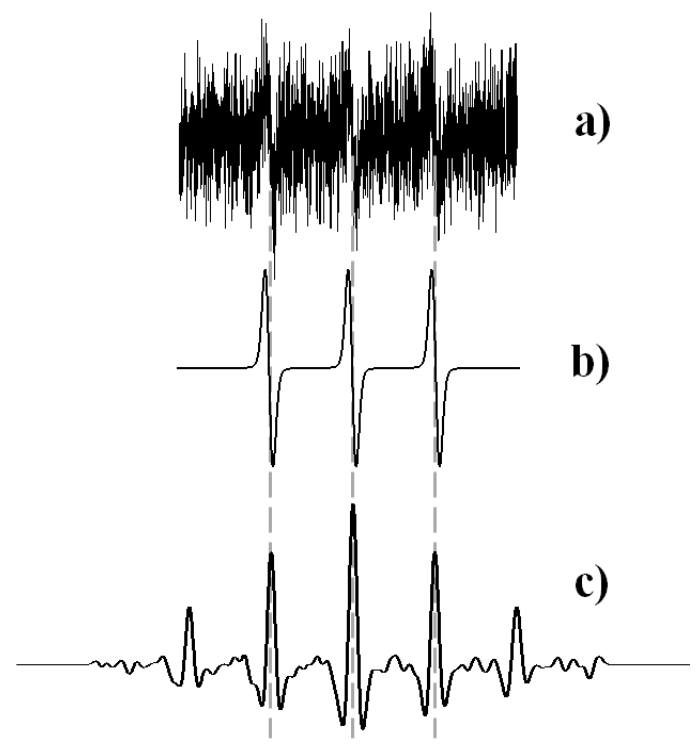

Figure 2. Electron paramagnetic resonance (EPR) spectrum of a liquid probe containing 4-hydroxy2,2,6,6-tetramethylpiperidine-1-oxide (TEMPOL) (a), the spectrum of TEMPOL in PBS (b), and the result of their convolution (c).

EPR spectra of nitroxides in swollen polymer samples are a combination of a broad EPR spectrum of corresponding radicals in dry polymer and a narrow triplet signal. To determine the contribution of the latter signal, the following procedures were used. If the narrow spectrum's intensity exceeded that of the broad spectrum, subtraction of the spectrum of the radicals in a dry polymer from the spectrum of the swollen sample was performed. Fourier transform of the swollen sample spectrum was used when the narrow spectrum intensity was low. The Fourier image's absolute value reveals a distinct periodic pattern arising from the narrow triplet signal (see Figure 3). This pattern can be described as follows [46]:

$$
\mathcal{F}\left[I_{E P R}\right](\omega)=C \cdot \omega \cdot \exp \left(-b_{G} \omega^{2}\right) \cdot \exp \left(-b_{L} \omega\right) \cdot \frac{|1+2 \cos (a \omega)|}{3}
$$


where $I_{E P R}$ is the EPR spectrum, $a$ is the hfi (hyper fine interaction) constant, $b_{G}$ and $b_{L}$ are constants depending on the spectrum shape, and $C$ is a relative integral contribution of the narrow triplet signal in the EPR spectrum.
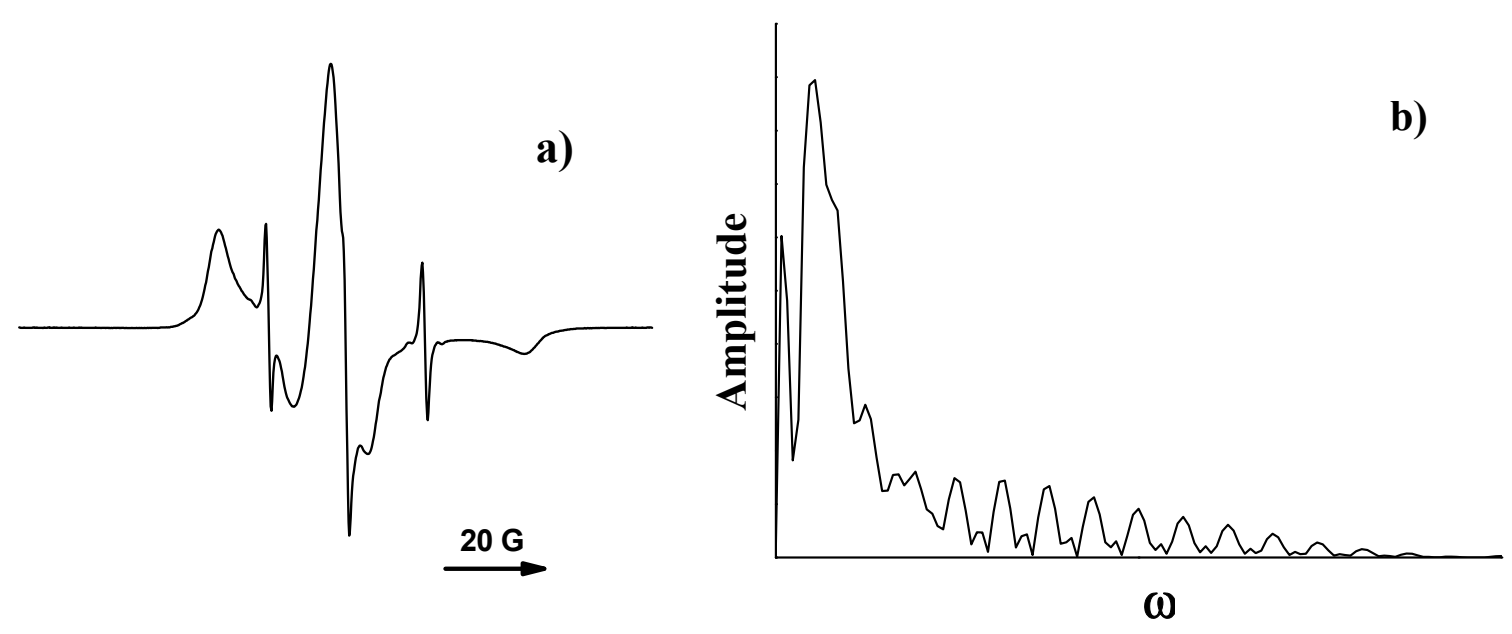

Figure 3. The EPR spectrum of 4-oxo-2,2,6,6-tetramethylpiperidine-1-oxide (TEMPONE) in swollen poly(D,L-lactide) film (a) and the absolute value of its Fourier image (b): the periodic pattern corresponds to the narrow triplet signal.

Fitting a periodic pattern of the Fourier image in the region corresponding to the narrow triplet signal allows for calculation of the contribution of this signal to the EPR spectrum of the swollen polymer film.

\section{Results and Discussion}

\subsection{Physicochemical Characterization of the Samples}

The number-average molecular weight $\left(M_{n}\right)$, weight-average molecular weight $\left(M_{w}\right)$, and polydispersity $(\mathrm{D})$ were determined by size exclusion chromatography using an Ultimate 3000 Thermo Scientific apparatus with Agilent PLgel $5 \mu \mathrm{m}$ MIXED-C $(300 \times 7.5 \mathrm{~mm})$ and one precolumn (PL gel $5 \mu \mathrm{m}$ guard $50 \times 7.5 \mathrm{~mm}$ ) thermostated at $30^{\circ} \mathrm{C}$. The detection was carried out using a differential refractometer as well as diode array detector. Tetrahydrofuran was eluted at a flow rate of $1.0 \mathrm{~mL} / \mathrm{min}$. The calculations of molecular weight and polydispersity were carried out using polystyrene standards (Agilent Technologies, Santa Clara, CA, USA). The obtained values of $M_{n}$ were then corrected by a factor of 0.58 [47]. The molecular weight characteristics of the polymers are presented in Table 1. It is seen that the proceeding of the polymers in $\mathrm{scCO}_{2}$ did not lead to significant changes in these parameters.

Table 1. Molecular weight characteristics of the polymers.

\begin{tabular}{cccc}
\hline & $\mathbf{M}_{\mathbf{n}}$ & $\mathbf{M}_{\mathbf{w}}$ & Ð \\
\hline PDL 04 & 14,800 & 36,100 & 2.4 \\
\hline $\begin{array}{c}\text { PDL 04, } \\
\text { after SCF processing }\end{array}$ & 15,200 & 35,800 & 2.4 \\
\hline PDL 02 & 7200 & 15,600 & 2.2 \\
\hline
\end{tabular}

In Figure 4, the SEM images of dry polymeric films are presented. It can be seen that the film is homogeneous at the micro level. 


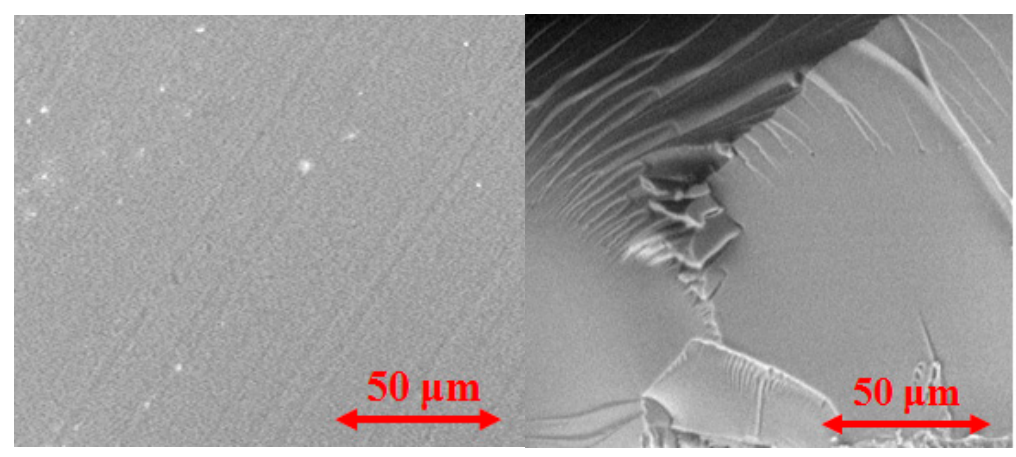

Figure 4. SEM images of dry $200 \mu \mathrm{m}$ poly(D,L-lactide) (PDL) 02 film: surface (left) and chip (right).

\subsection{Kinetic Regularities of the Dopants Release from the Poly(D,L-lactide) Films}

PR spectra of nitroxide radicals are triplets due to the hyperfine interaction of the unpaired electron with ${ }^{14} \mathrm{~N}$ nucleus (natural abundance $99.6 \%$, spin equals 1 ). Due to the high spatial anisotropy of hfc constant, the spectrum shape is susceptible to the rotational mobility of paramagnetic molecules [48]. Figure 5 shows the EPR spectra of TEMPOL in PBS at $298 \mathrm{~K}$ and in PDL 02 at $298 \mathrm{~K}$ and $100 \mathrm{~K}$. The spectra of all used radicals in PBS and within PDL 04 and PDL 02 are similar to the presented ones. It is seen that the spectra in poly (D,L-lactide) significantly differ from the spectrum in PBS. Because of the low mobility of radicals in the polymer matrix, the anisotropy of g- and hfc-tensors manifests in these spectra. It is necessary to note that the spectrum of the radicals in the polymer recorded at room temperature is close to that recorded at $100 \mathrm{~K}$ in the absence of rotational mobility of paramagnetic molecules (in the rigid limit). This fact indicates that the mobility of small compact TEMPOL molecules in PDL 02 is low (the rotational correlation time is about $10^{-7}-10^{-8} \mathrm{~s}$ ). The low mobility of dopant molecules shows that the free volume of PDLLA in glass state ( $T_{g}$ is approximately 323-326 K [49]) does not exceed the size of TEMPOL molecules ( $\mathrm{r}$ is approximately $3.4 \AA$ [50]). The spectrum of the radicals in PBS consists of three narrow lines because of averaging the magnetic anisotropy resulting from the rapid rotation of the paramagnetic molecules.

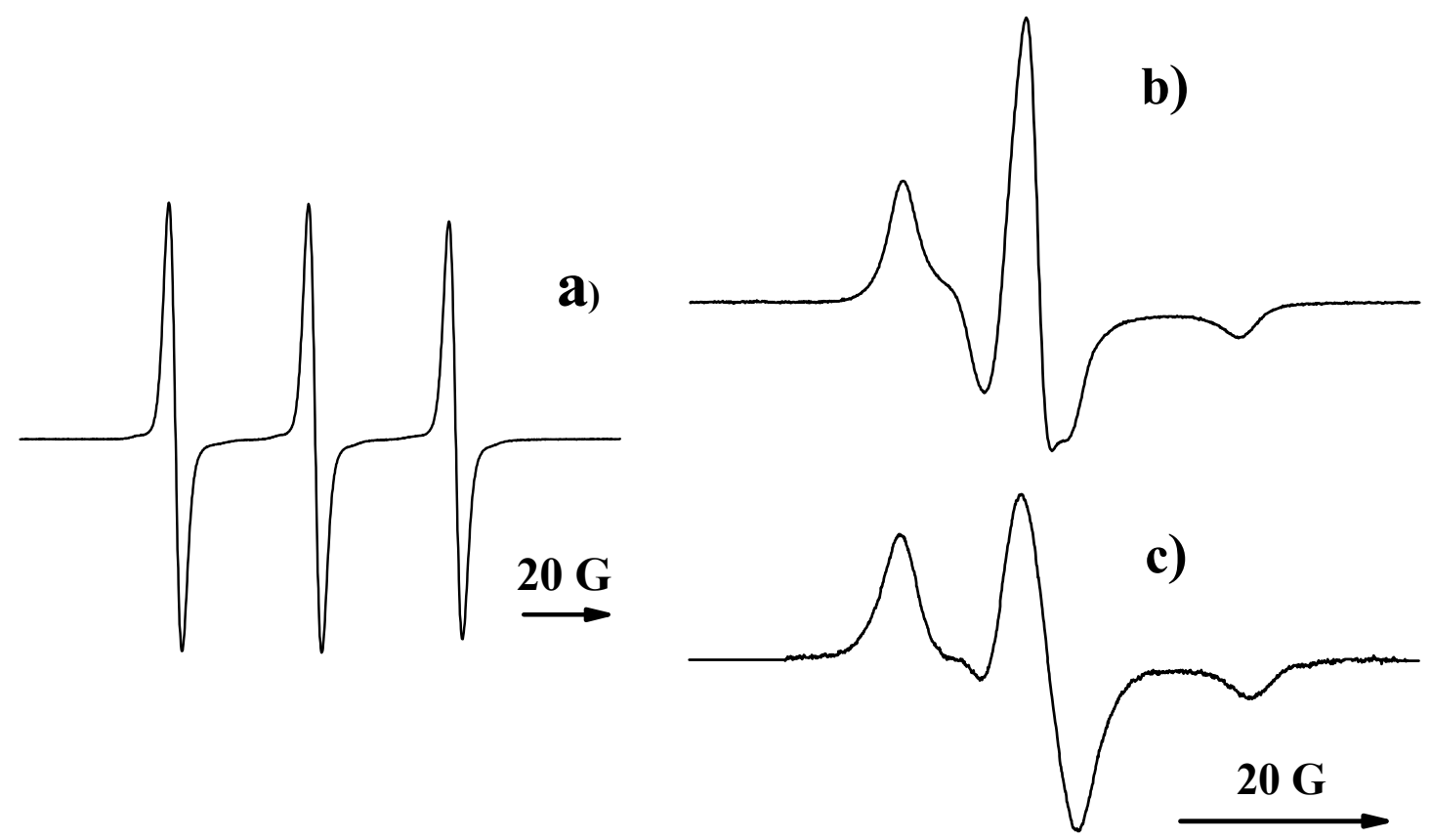

Figure 5. EPR spectra of TEMPOL in PBS at $298 \mathrm{~K}(\mathbf{a})$ and in PDL 02 at $298 \mathrm{~K}$ (b) and $100 \mathrm{~K}$ (c). 
One of the severe problems which can arise in the case of supercritical impregnation of a polymer is the nonuniform distribution of dopant molecules in the polymeric matrix. To determine local concentration of paramagnetic molecules, the approach proposed in [48] was used. The method is based on analysis of the broadening of the rigid limit spectrum due to the dipole-dipole interaction of paramagnetic molecules. It was found that the EPR spectra of all used paramagnetic substances in all formed samples are not broadened, so there is no reason to assume any significant irregularity of the radical's distribution in the polymer.

Figure 6 shows the kinetic curves corresponding to the release of different paramagnetic substances from PDL 02 and PDL 04 films into PBS. It is seen that the release of all studied dopants from PDL 02 started immediately after immersion of the samples into the liquid. In contrast, all dopants, regardless of their size and structure, stayed inside the PDL 04 film during ca. 90 days. Simultaneously, destruction of the PDL 04 samples was visually observed, so release of the dopant is most likely caused by erosion of the polymer. In several cases, a small amount (4-7\%) of radicals was released from PDL 04 during the first 5-7 days after sample immersion into PBS. Such an initial release (burst) was mentioned in many publications; the cause of this phenomenon is still being discussed $[28,51,52]$.
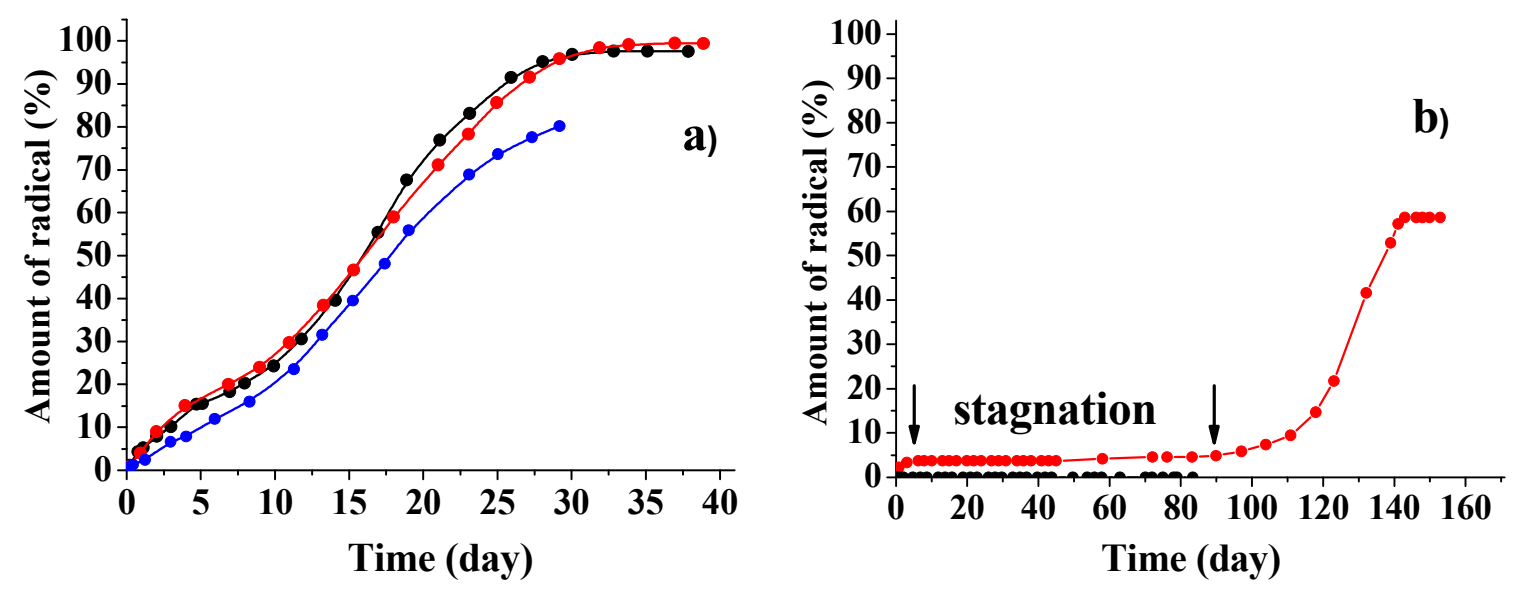

Figure 6. Kinetic curves corresponding to the release of TEMPOL (black and red symbols) and ATI (blue symbols) from PDL 02 films (a) and of sl-DCF (black symbols) and TEMPONE (red symbols) from PDL 04 films (b) into PBS: measurement errors are 10-12\%. The lines are not the result of fitting.

Several reasons can cause the observed essential difference in the kinetic regularities of the dopant release from PDL 02 and PDL 04 films; the most probable ones are the difference in the polymers' swelling rate and the pore formation processes. The rate of polymer swelling is characterized by the swelling index (SI), which is the ratio of water uptake to the mass of the dry sample. EPR spectroscopy provides an additional possibility of studying changes in the polymer structure during swelling and hydrolysis. Figure 7 shows the EPR spectra of TEMPOL in PDL 02 film recorded at different periods of swelling. It is seen that the spectra of the swollen samples are a combination of a broad EPR spectrum of radicals in dry polymer and a narrow triplet signal for which intensity increases with time. This signal is a spectrum of radicals localized in regions of the polymer into which water has penetrated. During the first $60 \mathrm{~min}$, the shape of the narrow triplet changes (Figure 7a). It seems that, in the initial period, a gradual increase in the probe mobility was caused by diffusion of water into the surface layer of the sample and gradual increase in the free volume of the polymer in swelling regions. Subsequently, the shape of the narrow triplet becomes close to that of the TEMPOL spectrum in PBS, so the main contribution to the triplet signal is made by paramagnetic molecules localized in pores filled with liquid. The numerical analysis reveals that, at all stages of swelling (except for the first $60 \mathrm{~min}$ ), the spectra can be presented as a sum of the radicals' spectrum in dry polymer and the narrow triplet signal. 

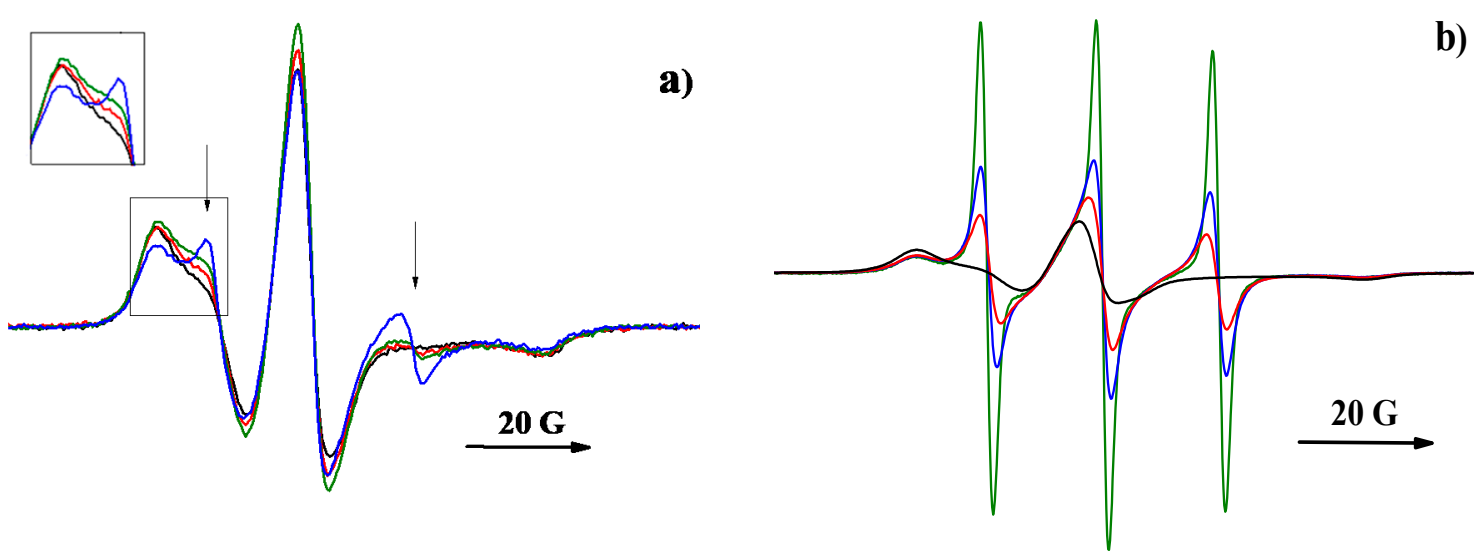

Figure 7. The EPR spectra of TEMPOL in PDL 02 film recorded at various holding times of the sample in solution: (a) dry sample (black line), $30 \mathrm{~min}$ (red line), $1 \mathrm{~h}$ (green line), and $6 \mathrm{~h}$ (blue line); (b) dry sample (black line), 2 days (red line), 7 days (blue line), and 17 days (green line).

Determination of the local $\mathrm{pH}$ inside the swollen polymer was performed using $\mathrm{pH}$-sensitive nitroxide radicals ATI and DPI. It is known that the distances between the components of the EPR spectra depend on the part of protonated paramagnetic molecules [40]. The calibration curve for ATI is published in [25], and that of DPI is given in Figure 8.

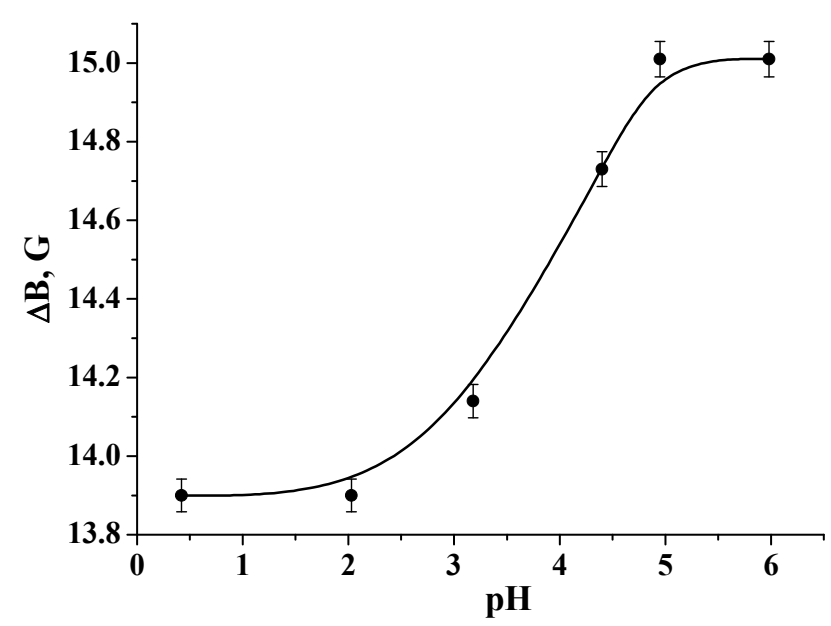

Figure 8. $\mathrm{pH}$ dependence of the distance between the central and left components $(\Delta \mathrm{B})$ of the EPR spectra of DPI in water solution.

It was found that local pH in the pores of PDL 02 and PDL 04 films was less than 2.5 from the start of the observation of the probe signal in the pores. Hence, we can conclude that most of the pores were formed due to autocatalytic hydrolysis of PDLLA. It is known that this process is accompanied by local acidification of the polymer matrix [32,53]. Figure 7 shows that ca. $60 \%$ of TEMPONE molecules release from PDL 04 film into PBS. The remaining ca. $40 \%$ of radicals convert into nonparamagnetic species due to disproportionation in acidified medium inside the swollen polymer's pores [54].

Figure 9 shows the time dependences of the polymers' swelling indexes and of the part of radicals localized in the pores. It is seen that both PDL 02 and PDL 04 swell in PBS, and the formation of the pores accompanies the swelling. The developed pore system was also visualized by optical microscopy (Figure 10). It is necessary to note that both PDL 02 and PDL 04 films become opaque after several hours in PBS. This fact confirms pore formation with a size close to the visible light wavelengths $(400-780 \mathrm{~nm})$. 

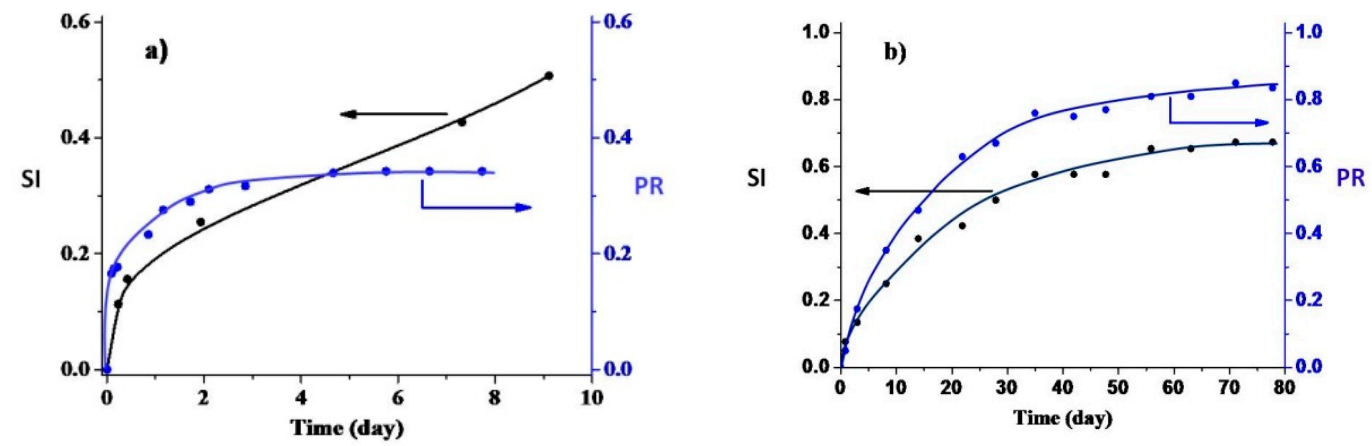

Figure 9. Time dependences of the swelling index (SI, black symbols, measurement errors are 5-7\%) and of the part of radicals, localized in pores (PR, blue symbols, measurement errors are 10-12\%) for TEMPOL in PDL 02 film (a) and for TEMPONE in PDL 04 film (b): the lines are not the results of fitting.

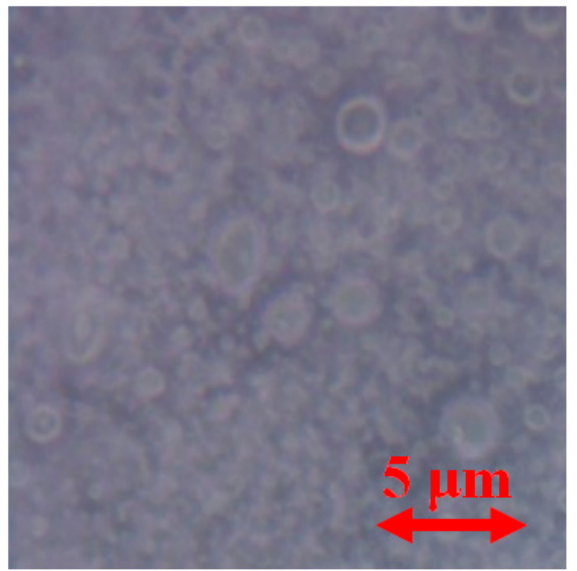

Figure 10. Optical microphotographs of PDL 04 film recorded after 3 weeks of keeping in PBS.

The swelling index and the part of the radicals localized in the pores of PDL 04 increase simultaneously during observation (Figure 9). These values for PDL 02 increase simultaneously during 4-5 days; after that, the swelling index increases, but the high mobile radicals' fraction remains constant. The dopant transition rate from the PDL 02 matrix to the pores after five days becomes equal to the dopant removal rate from the pores to the outer solution. Based on the above observations, one can assume that the pores in PDL 02 connect with the surface (open pores), while pores in PDL 04 do not (closed pores). It is in agreement with stagnation of the dopant release from PDL 04 for 90 days. SEM data confirm this assumption. The numerous pores connected with the surface arise in $2 \mathrm{~h}$ after immersion of the PDL 02 films into PBS (Figure 11). In contrast, the surface of PDL 04 films kept in PBS for 90 days remained smooth despite clearly recognizing the samples' swelling. On the 101st day, the areas containing numerous open pores can be seen on the PDL 04 film's surface. At that time, rapid release of the dopant was observed.

Considering the experimental facts, one can conclude that the transport of dopant molecules occurs basically through pores filled with liquid and connected with the sample's surface. To prove this hypothesis, the following experiments were performed. The fragments of PDL 04 films containing TEMPONE or sl-DCF were placed into PBS and in parallel to the solution of ascorbic acid (AA) in PBS. It is known that $\mathrm{AA}$ is one of the main reducing agents in living bodies which transforms nitroxide radicals to hydroxylamines [55]. AA is often used for investigation of nitroxide stability in different matrices $[56,57]$. Figure 12 shows that the radical's decomposition rate inside the swollen polymer does not depend on AA's presence in the outer liquid. Hence, the AA molecules do not penetrate inside the sample during swelling of the polymer. The AA concentration was chosen to be $5 \times 10^{-4} \mathrm{~g} / \mathrm{mL}$; $1 \%$ of it was sufficient to kill all paramagnetic molecules in the polymer. In contrast, the immersion of 
partially swollen PDL 02 film into the AA solution resulted in rapid decrease of triplet signal intensity. Obviously, in this case, AA diffused into the open pores and reacted with the nitroxides.
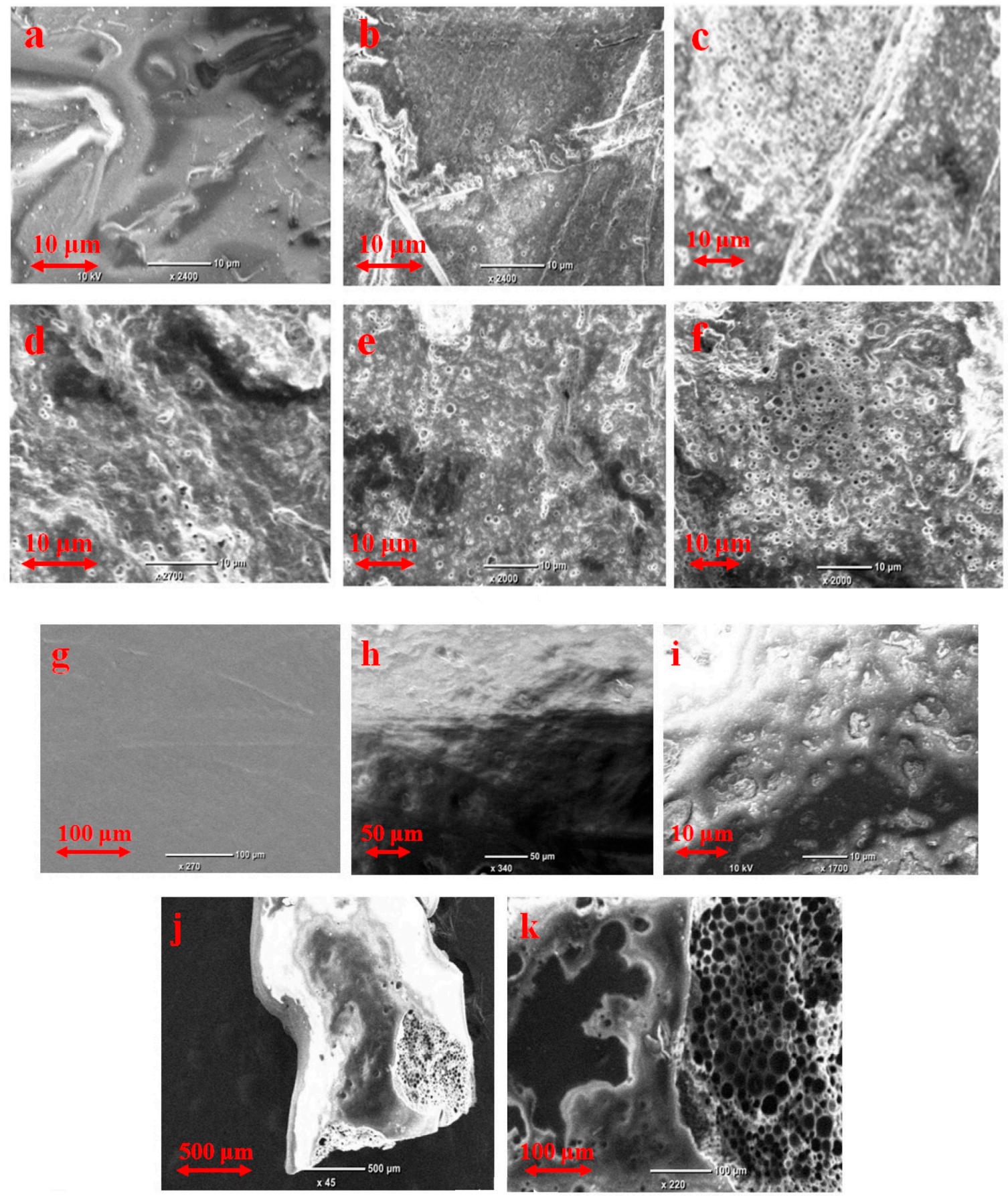

Figure 11. SEM microphotographs of PDL $02(\mathbf{a}-\mathbf{f})$ and PDL $04(\mathbf{g}-\mathbf{k})$ films recorded after different time of keeping them in PBS: (a) - dry film, (b) - $2 \mathrm{~h}$, (c) - 4 days, (d) -6 days, (e) -9 days, (f) -11 days, (g) -1 day, (h) - 5 days, (i) -90 days, and $(\mathbf{j}, \mathbf{k})-101$ days. 


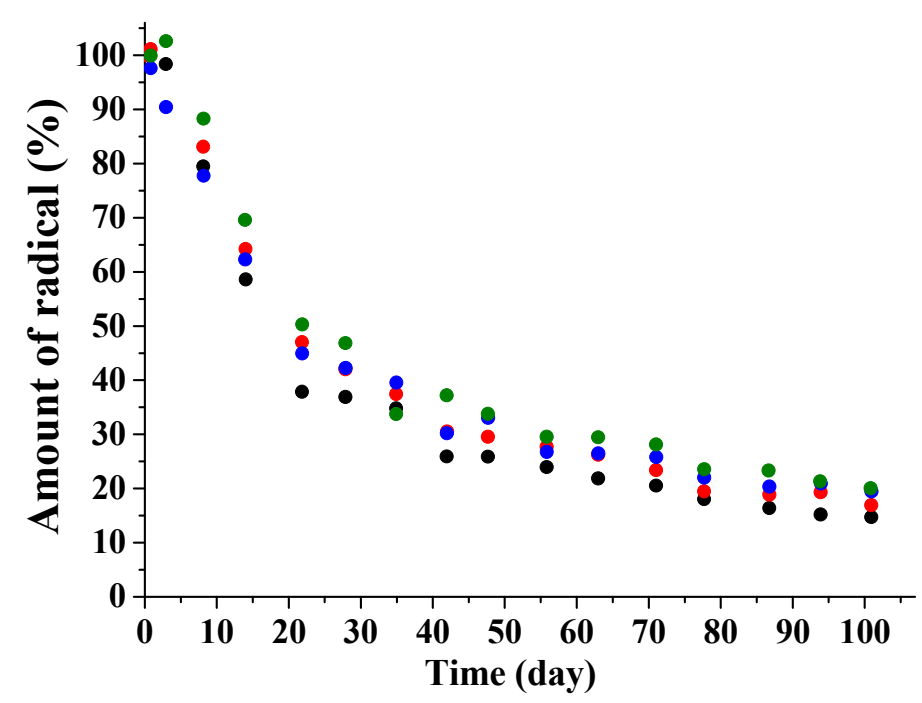

Figure 12. The kinetic curves of the radical's decomposition inside PDLLA films in the presence and in the absence of AA in the outer liquid: systems "TEMPONE/PBS + AA" (black symbols), "sl-DCF/PBS + AA" (red symbols), “TEMPONE/PBS” (blue symbols), and "sl-DCF/PBS” (green symbols). Measurement errors are $10-12 \%$.

\subsection{Mathematical Modeling of the Release Process}

The development of a mathematical model describing the kinetics regularities of the dopant release from PDLLA into aqueous media is one of the most critical challenges in understanding the mechanism of biodegradation. A reliable model would allow the creation of materials with the required characteristics. The development of the mathematical model turned out to be quite tricky because of many processes that coincide during swelling and degradation of the polymer, such as diffusion of water, formation and evolution of pores, hydrolysis of ester bonds, and diffusion of a dopant through the polymer matrix and in its pores, etc. To date, most of the experimental results concerning the change of polylactide structure during swelling and hydrolysis were obtained using chromatography analysis that is the molecular weight change control [29,58,59]. For this reason, most of the mathematical models describing the dopant release from polylactide to the aqueous medium are based on the diffusion of dopant molecules through swollen and partially hydrolyzed polymer matrices $[26,60,61]$. Our experiments unambiguously show that diffusion of the dopant molecules is mainly caused by dopant release through the pores filled with liquid not through the polymer matrix. Hence, the mathematical model of the release regularities should be based on formation of the pores.

In the present paper, we announce the mathematical model of a dopant release from PDL 02 films. In this case, the release is controlled by swelling and hydrolysis of the polymer unlike PDL 04, where the release is controlled by erosion of the polymer matrix. Figure 13 shows the differential kinetic curves of the dopant release from PDL 02 films normalized to the initial content of the paramagnetic substance in the samples. For further analysis, the kinetic curves were approximated by polynomials of 5-6 degrees.

The kinetic curve for the $50 \mu \mathrm{m}$ sample has no notable features (Figure 13b). The kinetic curves for the $200 \mu \mathrm{m}$ samples are functions with extrema; they have both minimum and maximum. The minimum observed on 5-7 days indicates several processes; one of them leads to a slowdown, and the other one leads to an acceleration of the release rate. The maximum observed at 17-18 days is apparently due to the accelerated release of the dopant and simultaneous decrease in the total amount of the paramagnetic molecules inside the polymer due to their irreversible removal from the system. 

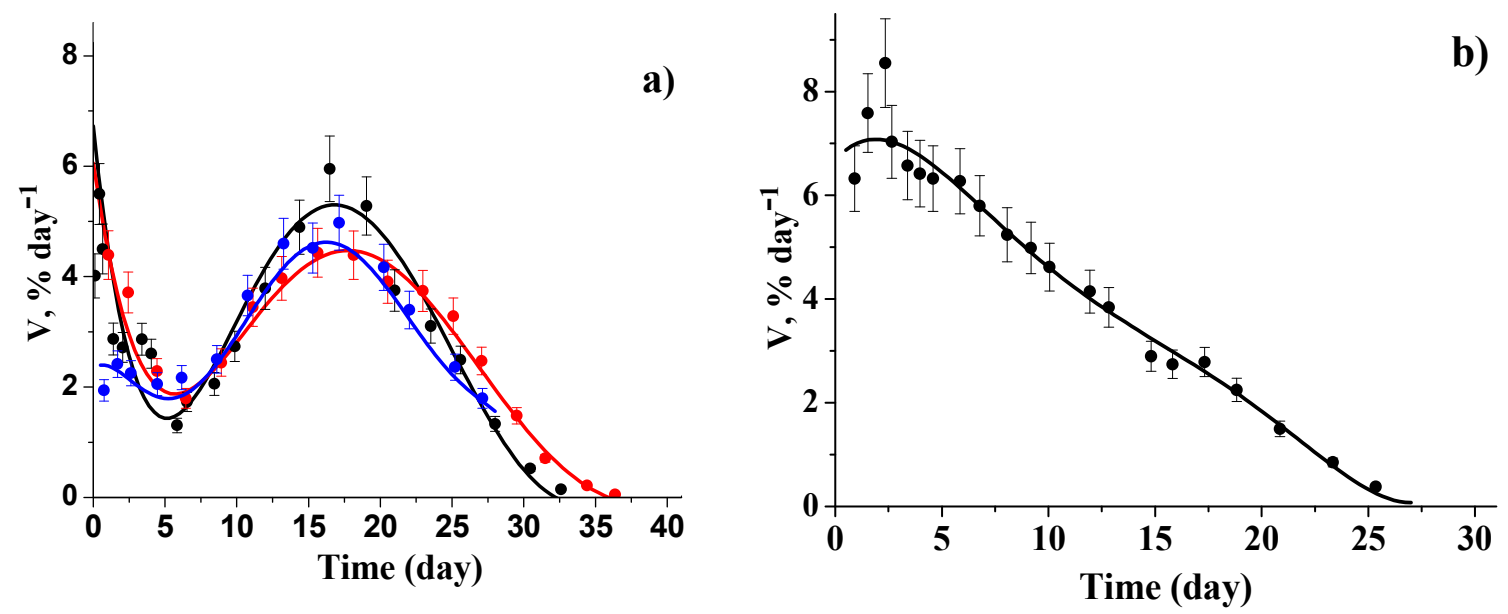

Figure 13. Differential kinetic curves for the dopants release from PDL 02 films: TEMPOL/200 $\mu \mathrm{m}$ films (black and red symbols) and ATI/200 $\mu \mathrm{m}$ film (blue symbols) (a), and TEMPOL/50 $\mu \mathrm{m}$ film (b). The lines are the result of polynomial fitting.

Thorough analysis of the SEM images of the PDL 02 films surface allows for establishment of a reason for the minimum on the kinetic curves. Table 2 shows the average number of pores on the surface corresponding to different sample holding times in liquid. It is seen that, after six days, the concentration of surface pores decreased. After that, on days 9 and 11, many pores connected with the surface and characterized by a complex internal structure appeared. The pore closing (pore healing) on the surface of aliphatic polyesters after keeping them in an aqueous medium is discussed in the literature $[51,62,63]$. This phenomenon is explained by local plasticization caused by penetration of water into the near-surface area of the polymer. Optical microscopy revealed a significant number of non-surface (closed) pores inside PDL 02 film, which was kept in PBS for six days. A large number of opened (connected with the surface) pores on 9-11 days, apparently, were formed by the connection of the closed pores with the surface of the film. Based on the observations, two stages of formation of the pores can be indicated. Therefore, the dopant release can be divided into two phases: release through surface pores, which are partially closed with time, and release through pores formed inside the polymer matrix due to autocatalytic hydrolysis of the polymer and gradually connected to the surface of the sample. For some time, these processes cooccur.

Table 2. An average number of pores on the surface of the swelling PDL 02.

\begin{tabular}{cc}
\hline Time & Average Number of Pores per $\mathbf{1 0 0} \boldsymbol{\mu m}^{\mathbf{2}}$ \\
\hline $2 \mathrm{~h}$ & 16 \\
2 days & 7 \\
4 days & 8 \\
6 days & 4 \\
9 days & 8 \\
11 days & 10 \\
\hline
\end{tabular}

Mathematical modeling of the first stage of the release is a difficult task because of the simultaneous occurrence of many processes-diffusion of water into the polymer, hydrolysis of polymer chains, diffusion of soluble oligomers to the outside, formation and growth of pores, closure of part of the pores on the surface, etc. Many authors have proposed approaches describing these processes separately [59,64-67], but the unified mathematical model has not been worked out. We describe the first stage release as Fick diffusion without detailed consideration of each process. To account for 
changes in the polymer matrix during swelling and hydrolysis, we introduce the diffusion coefficient's dependence on time and coordinate $D_{I}(x, t)$ :

$$
D_{I}(x, t)=D_{0} \exp \left(-\lambda\left(\frac{L}{2}-|x|\right)\right)\left(1-f \cdot e^{-\mu t}\right)
$$

where $L$ is the film thickness, $x$ is the distance between the point under consideration and the center of the film, and subscript $I$ implies the first stage of the release process. Parameters $D_{0}, \lambda, f, \mu$ characterize the processes that occur in the early stages of polymer swelling and hydrolysis. Since some of the pores which were formed at the first stage do not close but continue to grow deep into the sample, dependence (2) was taken into account when describing the experiment over the entire time.

Modeling the release kinetics at the second stage was performed using a diffusion model proposed in [31] and based on the formation of pores connected with the surface and filled with liquid. According to this model, the dopant molecules diffuse through the polymer matrix with a diffusion coefficient $D_{S}$, penetrate the pores, and diffuse in the liquid inside the pores with a diffusion coefficient $D_{L}$. Distribution of the low-molecular substance between the liquid phase located in the pores and the polymer phase is described by the distribution constant.

$$
\kappa=\frac{C_{L}(x, t)}{C_{S}(x, t)}=\text { const }
$$

The latter hypothesis was not studied experimentally in [31], but our experiments' results confirm its reliability. One of the basic parameters of the model [31] is the porosity function $\varphi(t)$ which is the volume fraction of the pores formed at a particular point in time. According to our experimental observations, this function should be interpreted as the volume fraction of the pores connected with the sample's surface (open pores). In [31], the porosity function was presented as follows:

$$
\varphi(t)=\varphi_{0}+\left(1-\varphi_{0}\right)\left(1-e^{-k t}\right)^{2}
$$

where $\varphi_{0}$ is the initial porosity; thus, the formation of pores begins immediately after immersion of the sample in an aqueous medium. To describe the second stage of the release, the porosity function should be shifted in time. Besides, to take into account the hydrolysis's autocatalytic character, we changed the rate of the function increase by changing the exponent:

$$
\varphi(t)=\left\{\begin{array}{c}
0, t<t_{\text {lag }} \\
\left(1-e^{-k\left(t-t_{\text {lag }}\right)}\right)^{\frac{3}{2}}, t>t_{\text {lag }}
\end{array} .\right.
$$

where $t_{\text {lag }}$ means the time when the second stage of release starts.

Penetration of dopant molecules into the pores and dopant release as these pores gradually connects with the surface are described in [68] by the average diffusion coefficient:

$$
D_{I I}(t)=\frac{(1-\varphi(t)) D_{S 0} e^{\alpha k_{W^{t}}}+\kappa \varphi(t) D_{L}}{1-\varphi(t)+\kappa \varphi(t)}
$$

where $D_{L}$ is the effective diffusion coefficient of the dopant in the pore which also reflects complex geometry of the pores, $D_{S 0}$ is the diffusion coefficient of the dopant in a dry polymer, $k_{W}$ is the rate constant of hydrolysis of the polymer chains, $\alpha$ is the empirical parameter connecting the diffusion coefficient of the dopant in the polymer matrix with the average molecular weight of the polymer, and subscript II means the second stage of the release process.

Under the conditions of our experiments, the contribution of the dopant diffusing through the polymer matrix to the release process is significantly lower (1-2 order of magnitude) than the contribution of the dopant diffusing through the pores. Therefore, the summand $D_{S}(t)=D_{S 0} e^{\alpha k_{W} t}$ can 
be neglected. The values of $\varphi(t)$ turn out to be relatively small throughout the experiment; therefore, we can only consider the first term in the Taylor series:

$$
\begin{gathered}
\varphi(t)=\left(1-e^{\left.-k\left(t-t_{\text {lag }}\right)\right)^{\frac{3}{2}}} \approx\left(k\left(t-t_{\text {lag }}\right)\right)^{\frac{3}{2}}=k^{\frac{3}{2}}\left(t-t_{\text {lag }}\right)^{\frac{3}{2}}, t>t_{\text {lag }}\right. \\
D_{\text {II }}(t)=\frac{\kappa D_{L} k^{\frac{3}{2}}\left(t-t_{\text {lag }}\right)^{\frac{3}{2}}}{1-\varphi(t)+\kappa \varphi(t)} \approx \kappa D_{L} k^{\frac{3}{2}}\left(t-t_{\text {lag }}\right)^{\frac{3}{2}}, t>t_{\text {lag }} .
\end{gathered}
$$

From Expression (7), it is seen that the term $A=\kappa D_{L} k^{3 / 2}$ should be considered a unified parameter. When developing the mathematical model, the following additional assumptions were taken into account:

(1) The dry polymer film is structurally uniform.

(2) The dopant is distributed uniformly in a dry sample.

(3) Diffusion through the side surface of the film is negligible.

(4) Perfect-sink conditions are used.

Based on the assumptions described above, the mathematical model has the following form.

$$
\begin{gathered}
\frac{\partial C(x, t)}{\partial t}=\frac{\partial}{\partial x}\left[D(x, t) \frac{\partial C(x, t)}{\partial x}\right] \\
D(x, t)=D_{0} \exp \left(-\lambda\left(\frac{L}{2}-x\right)\right)\left(1-f e^{-\mu t}\right)+\kappa D_{L} \varphi(t) \\
C(x, 0)=C_{0} \\
C\left(\frac{L}{2}, t\right)=0 \\
\frac{\partial C(x, t)}{\partial x} x_{x=0}=0 \\
x \in\left[0, \frac{L}{2}\right], t \in\left[0, t_{\text {max }}\right]
\end{gathered}
$$

After solving the differential equation, the release rate normalized to the initial amount of dopant in the sample $\left(N_{0}\right)$ can be calculated as follows:

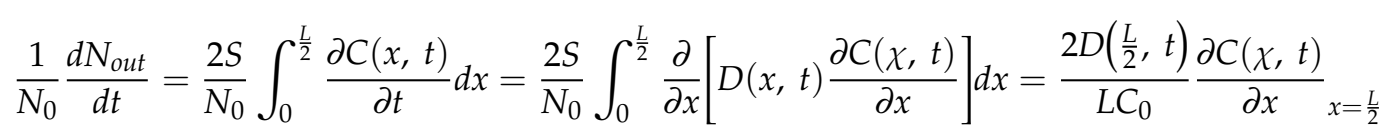

On the whole, the diffusion model is characterized by the following parameters: $D_{0}, \lambda, \mu$, and $f$ are empirical parameters describing the first stage release; $t_{\text {lag }}$ defines the time when connection of the internal pores with the surface starts; and parameter $A=\kappa D_{L} k^{3 / 2}$ reflects the intensity of dopant release at the second stage. The last parameter characterizes the affinity of the dopant for the matrix and the hydrolytic stability of the matrix.

The results of modeling the experimental differential kinetic curves are presented in Figure 14. Figure 15 shows the corresponding descriptions of the integral kinetic curves. It is seen that, despite a slight deviation of the theoretical differential curves from the experimental ones, the description of the integral release curves is within the registration errors. The results indicate that the difference in the shape of the kinetic curves for $50 \mu \mathrm{m}$ and $200 \mu \mathrm{m}$ films is due to different contributions of the dopant release through the pores formed in the near surface layer (first stage of pore formation) and through the pores formed inside the polymer and then connected with the surface (second stage of pore formation). 

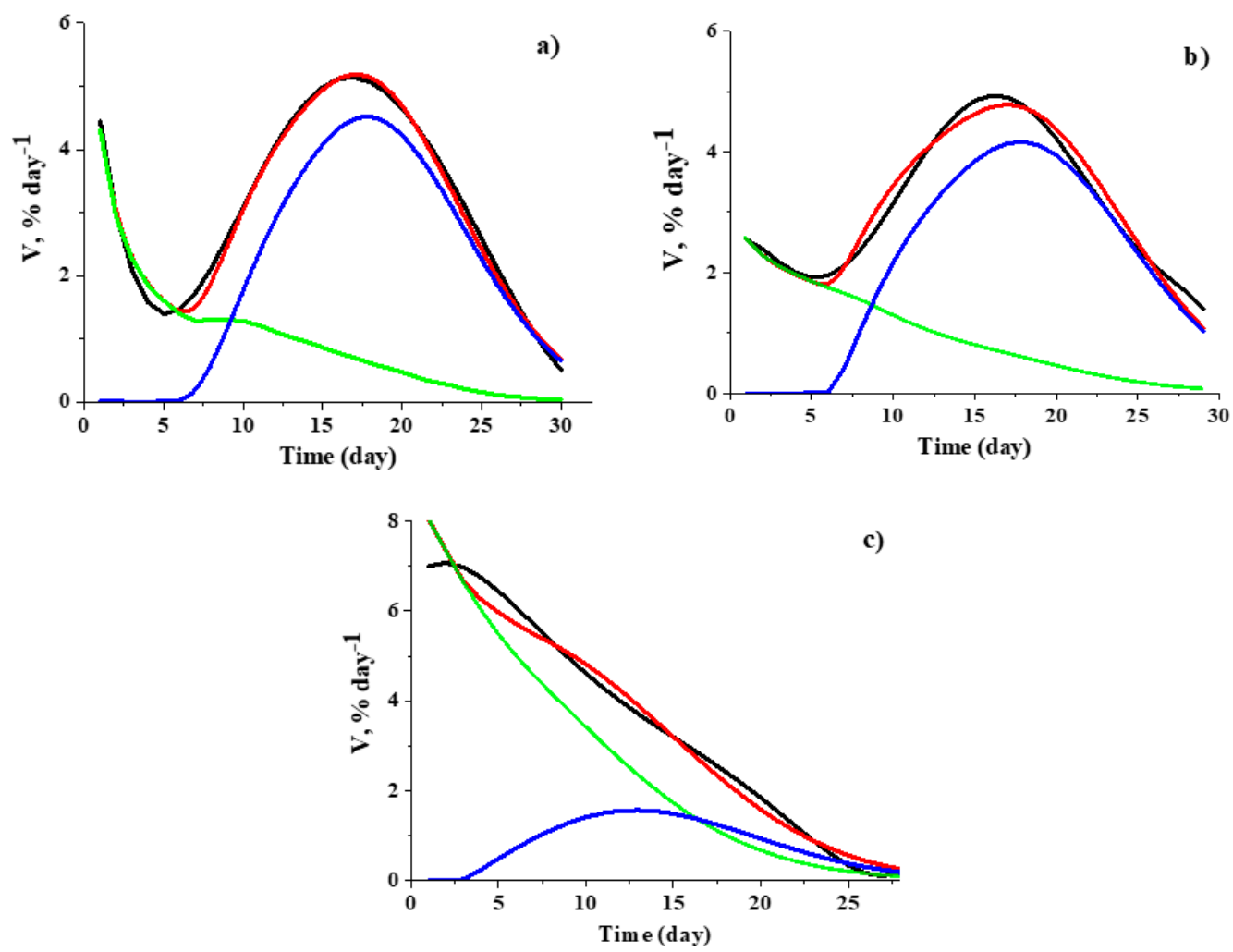

Figure 14. Experimental differential curves (black lines) and corresponding numerical modeling of TEMPOL (a) and ATI (b) release from $200 \mu \mathrm{m}$ films and of TEMPOL (c) release from $50 \mu \mathrm{m}$ film: the red lines are the results of modeling, and green and blue lines are contributions of the dopant release through the pores formed at the first and the second stages of pore formation, respectively (see text).
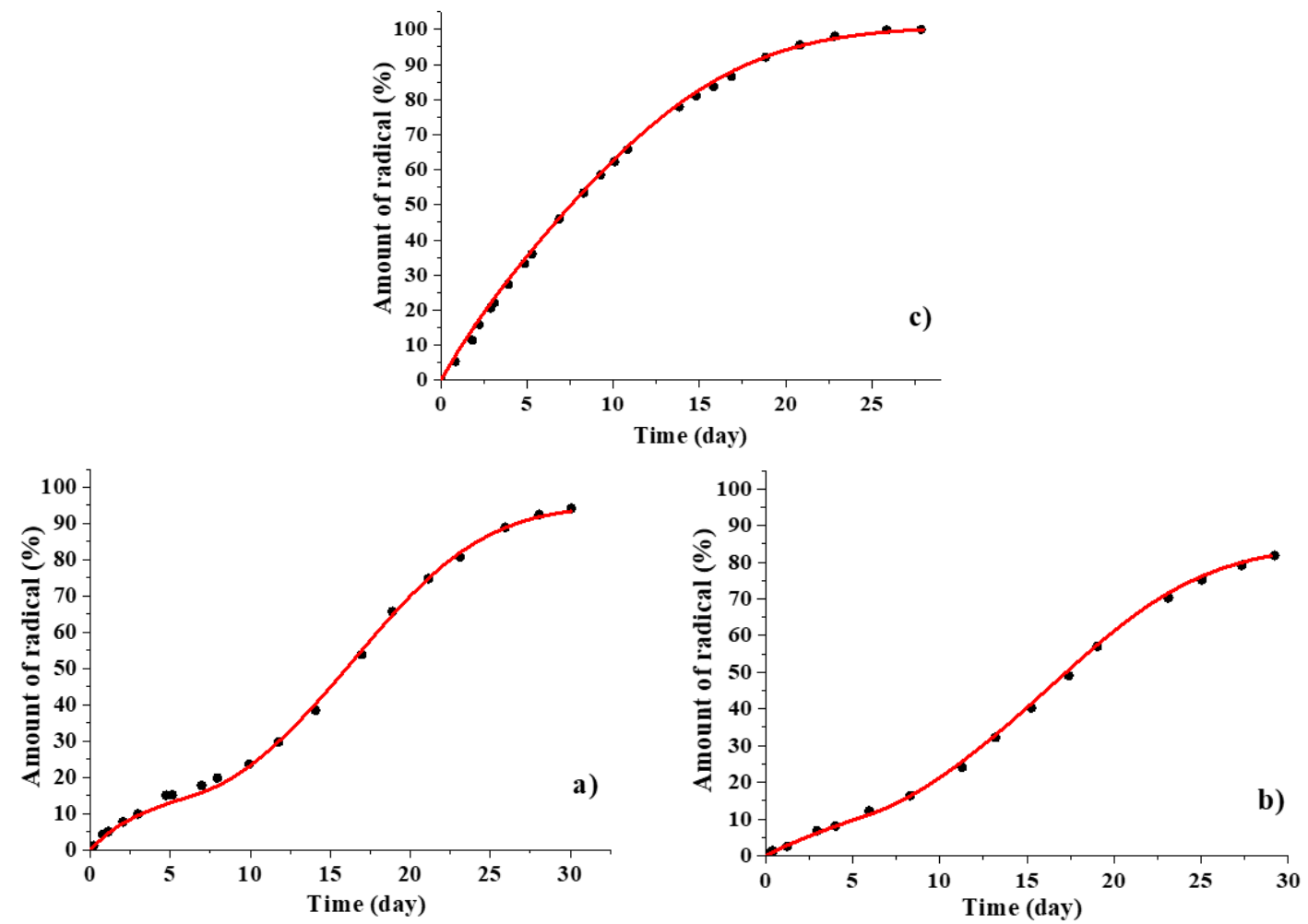

Figure 15. Experimental cumulative curves (dots) and corresponding numerical modeling (lines) of TEMPOL (a) and ATI (b) release from $200 \mu \mathrm{m}$ PDLLA films and of TEMPOL (c) release from $50 \mu \mathrm{m}$ film. 
The parameters of the modeling are presented in Table 3. For system 1, two independent release experiments were performed. It can be noted that the optimal value of parameter $t_{\text {lag }}$ for system 1 is close to the time when the fraction of mobile radicals reached the limit (Figure 9) and is consistent with the data obtained by scanning electron microscopy (Figure 11). The value of parameter $A$ for $200 \mu \mathrm{m}$ films is approximately 20 times higher than for $50 \mu \mathrm{m}$ film. Since the value $\kappa$ contributing to this parameter is determined only by the nature of the polymer and dopant, the observed difference reflects greater hydrolytic stability of thin PDLLA films, consistent with published data [68-70]. Parameter $D_{0}$ reflects the rate of pore formation at the first stage. One can assume from the data of Table 3 a slower surface pore formation in a thin film. The absence of correlation of the parameters $\lambda, \mu$, and $f$ both with film thickness and the nature of the radical perhaps indicates differences in the structure of the surface layer caused by manual pressing of the films. Further application of the proposed to various "polymer/dopant" systems would clarify these parameters' physical meaning.

Table 3. The optimal values of the modeling parameters.

\begin{tabular}{|c|c|c|c|c|c|c|c|}
\hline & System & $\mathrm{D}_{0}, \mathrm{~m}^{2} \cdot \mathrm{Day}^{-1}$ & $\lambda, \mathrm{m}^{-1}$ & $\mu$, Day $^{-1}$ & $f$ & $t_{\text {lag, }}$ Day & A, m ${ }^{2} \cdot$ Day $^{-5 / 2}$ \\
\hline 1 & TEMPOL/200 $\mu \mathrm{m}$ & $7.5 \times 10^{-11}$ & $5.0 \times 10^{4}$ & 1.4 & 0.5 & 6.1 & $1.35 \times 10^{-11}$ \\
\hline 1 & TEMPOL/200 $\mu \mathrm{m}$ & $7.5 \times 10^{-11}$ & $2.5 \times 10^{4}$ & 1.0 & 0.3 & 5.9 & $0.94 \times 10^{-11}$ \\
\hline 2 & TEMPOL/50 $\mu \mathrm{m}$ & $2.4 \times 10^{-11}$ & $0.9 \times 10^{4}$ & 0.33 & 1.0 & 2.6 & $4.77 \times 10^{-13}$ \\
\hline 3 & ATI/200 $\mu \mathrm{m}$ & $6.9 \times 10^{-11}$ & $1.4 \times 10^{4}$ & 0.17 & 0.9 & 5.8 & $1.11 \times 10^{-11}$ \\
\hline
\end{tabular}

\section{Conclusions}

EPR spectroscopy combined with modern methods of numerical analysis of spectra allowed for monitoring of the changes in the paramagnetic probe content both in the biodegradable polymeric matrix during hydrolysis and in the external environment simultaneously. As a result, it was found that loss of the probe inside the poly(D,L-lactide) did not mean only its transition to the external environment. In an acidic medium formed during hydrolysis of the polyester, radicals may transform into diamagnetic substances. Registration of the release curves in a differential form allowed for estimation of the pronounced extrema of the release rate on time, making it possible to narrow the range of mathematical models of the release. Joint analysis of EPR spectroscopy, SEM, and water uptake data allowed us to conclude that the central role in the release plays diffusion in pores that arise, grow, or close up in the polymer matrix. Combination and competition of these processes explain the "burst" and lag periods. It was proposed that diffusion through the swollen polymer is negligible. The mathematical model of the release kinetics based on pore formation was performed.

Author Contributions: Conceptualization and methodology, N.A.C. and E.N.G.; software and formal analysis, S.V.K. (Sergei V. Kuzin); investigation, S.V.K. (Sergei V. Kuzin), T.A.I., and S.V.K. (Sergey V. Kostjuk); resources, I.A.G.; writing - original draft preparation, N.A.C.; writing—review and editing, E.N.G.; project administration, M.Y.M. All authors have read and agreed to the published version of the manuscript.

Funding: This work was supported by the Russian Foundation for Basic Research (grant 18-29-06059), by the Russian Science Foundation (grant 19-13-00235 in part of spin probes synthesis), and in part by M.V. Lomonosov Moscow State University Program of Development.

Acknowledgments: The authors acknowledge O.I. Gromov for assistance in polymer impregnation in supercritical $\mathrm{CO}_{2}$, T. Kalai (University of Pécs, Hungary) for providing us with spin-labeled bioactive diclofenac (sl-DCF), and S.N. Churbanov for assistance in film preparation.

Conflicts of Interest: The authors declare no conflict of interest.

\section{References}

1. Hench, L.L.; Jones, J.R. Biomaterials, Artificial Organs and Tissue Engineering; Elsevier: Amsterdam, The Netherlands, 2005; ISBN 9781855737372.

2. Giandalia, G.; De Caro, V.; Cordone, L.; Giannola, L.I. Trehalose-hydroxyethylcellulose microspheres containing vancomycin for topical drug delivery. Eur. J. Pharm. Biopharm. 2001, 52, 83-89. [CrossRef] 
3. Chrzanowska, O.; Struszczyk, M.H.; Krucinska, I.; Puchalski, M.; Herczyńska, L.; Chrzanowski, M. Elaboration of small-diameter vascular prostheses-Selection of appropriate sterilisation method. J. Appl. Polym. Sci. 2014, 131, 9611-9620. [CrossRef]

4. Gentile, P.; Chiono, V.; Carmagnola, I.; Hatton, P. An Overview of Poly(lactic-co-glycolic) Acid (PLGA)-Based Biomaterials for Bone Tissue Engineering. Int. J. Mol. Sci. 2014, 15, 3640-3659. [CrossRef] [PubMed]

5. Stewart, S.; Domínguez-Robles, J.; Donnelly, R.; Larrañeta, E. Implantable Polymeric Drug Delivery Devices: Classification, Manufacture, Materials, and Clinical Applications. Polymers 2018, 10, 1379. [CrossRef] [PubMed]

6. Chang, L.; Liu, J.; Zhang, J.; Deng, L.; Dong, A. pH-sensitive nanoparticles prepared from amphiphilic and biodegradable methoxy poly(ethylene glycol)-block-(polycaprolactone-graft-poly(methacrylic acid)) for oral drug delivery. Polym. Chem. 2013, 4, 1430-1438. [CrossRef]

7. Montes, A.; Gordillo, M.D.; Pereyra, C.; De los Santos, D.M.; Martínez de la Ossa, E.J. Ibuprofen-polymer precipitation using supercritical $\mathrm{CO}_{2}$ at low temperature. J. Supercrit. Fluids 2014, 94, 91-101. [CrossRef]

8. Elvira, C.; Fanovich, A.; Fernández, M.; Fraile, J.; San Román, J.; Domingo, C. Evaluation of drug delivery characteristics of microspheres of PMMA-PCL-cholesterol obtained by supercritical- $\mathrm{CO}_{2}$ impregnation and by dissolution-evaporation techniques. J. Control. Release 2004, 99, 231-240. [CrossRef]

9. Mashak, A.; Mobedi, H.; Mahdavi, H. A Comparative Study of Progesterone and Lidocaine Hydrochloride Release from Poly(L-lactide) Films. Pharm. Sci. 2015, 21, 77-85. [CrossRef]

10. Ramchandani, M.; Robinson, D. In vitro and in vivo release of ciprofloxacin from PLGA 50:50 implants. J. Control. Release 1998, 54, 167-175. [CrossRef]

11. Cabezas, L.I.; Fernández, V.; Mazarro, R.; Gracia, I.; De Lucas, A.; Rodríguez, J.F. Production of biodegradable porous scaffolds impregnated with indomethacin in supercritical $\mathrm{CO}_{2}$. J. Supercrit. Fluids 2012, 63, 155-160. [CrossRef]

12. Guney, O.; Akgerman, A. Synthesis of controlled-release products in supercritical medium. AIChE J. 2002, 48, 856-866. [CrossRef]

13. Park, M.W.; Bae, H.K. Dye distribution in supercritical dyeing with carbon dioxide. J. Supercrit. Fluids 2002, 22, 65-73. [CrossRef]

14. Nazem, N.; Taylor, L.T.; Rubira, A.F. Metallized poly(etherether ketone) films achieved by supercritical fluid impregnation of a silver precursor followed by thermal curing. J. Supercrit. Fluids 2002, 23, 43-57. [CrossRef]

15. Said-Galiyev, E.; Nikitin, L.; Vinokur, R.; Gallyamov, M.; Kurykin, M.; Petrova, O.; Lokshin, B.; Volkov, I.; Khokhlov, A.; Schaumburg, K. New chelate complexes of copper and iron: Sythesis and impregnation into a polymer matrix from solution in supercritical carbon dioxide. Ind. Eng. Chem. Res. 2000, 39, 4891-4896. [CrossRef]

16. Li, D.; Liu, Z.; Han, B.; Song, L.; Yang, G.; Jiang, T. Preparation of nanometer dispersed polypropylene/polystyrene interpenetrating network using supercritical $\mathrm{CO}_{2}$ as a swelling agent. Polymer 2002, 43, 5363-5367. [CrossRef]

17. Tang, M.; Wen, T.-Y.; Du, T.-B.; Chen, Y.-P. Synthesis of electrically conductive polypyrrole-polystyrene composites using supercritical carbon dioxide. Eur. Polym. J. 2003, 39, 143-149. [CrossRef]

18. Fu, Y.; Kao, W.J. Drug release kinetics and transport mechanisms of non-degradable and degradable polymeric delivery systems. Expert Opin. Drug Deliv. 2010, 7, 429-444. [CrossRef]

19. Strobel, C.; Bormann, N.; Kadow-Romacker, A.; Schmidmaier, G.; Wildemann, B. Sequential release kinetics of two (gentamicin and BMP-2) or three (gentamicin, IGF-I and BMP-2) substances from a one-component polymeric coating on implants. J. Control. Release 2011, 156, 37-45. [CrossRef]

20. Dong, Y.; Zhang, Z.; Feng, S.-S. d- $\alpha$-Tocopheryl polyethylene glycol 1000 succinate (TPGS) modified poly(l-lactide) (PLLA) films for localized delivery of paclitaxel. Int. J. Pharm. 2008, 350, 166-171. [CrossRef]

21. Shen, E.; Kipper, M.J.; Dziadul, B.; Lim, M.K.; Narasimhan, B. Mechanistic relationships between polymer microstructure and drug release kinetics in bioerodible polyanhydrides. J. Control. Release 2002, 82, 115-125. [CrossRef]

22. Molavi, F.; Barzegar-Jalali, M.; Hamishehkar, H. Polyester based polymeric nano and microparticles for pharmaceutical purposes: A review on formulation approaches. J. Control. Release 2020, 320, 265-282. [CrossRef] [PubMed] 
23. Ritger, P.L.; Peppas, N.A. A simple equation for description of solute release I. Fickian and non-fickian release from non-swellable devices in the form of slabs, spheres, cylinders or discs. J. Control. Release 1987, 5, $23-36$. [CrossRef]

24. Ford, J.L.; Mitchell, K.; Rowe, P.; Armstrong, D.J.; Elliott, P.N.C.; Rostron, C.; Hogan, J.E. Mathematical modelling of drug release from hydroxypropylmethylcellulose matrices: Effect of temperature. Int. J. Pharm. 1991, 71, 95-104. [CrossRef]

25. Kao, C.-C.; Chen, S.-C.; Sheu, M.-T. Lag time method to delay drug release to various sites in the gastrointestinal tract. J. Control. Release 1997, 44, 263-270. [CrossRef]

26. Raman, C.; Berkland, C.; Kim, K.; Pack, D.W. Modeling small-molecule release from PLG microspheres: Effects of polymer degradation and nonuniform drug distribution. J. Control. Release 2005, 103, 149-158. [CrossRef] [PubMed]

27. Lindner, W.D.; Lippold, B.C. Drug Release From Hydrocolloid Embeddings with High or Low Susceptibility to Hydrodynamic Stress. Pharm. Res. Off. J. Am. Assoc. Pharm. Sci. 1995, 12, 1781-1785.

28. Huang, X.; Brazel, C.S. On the importance and mechanisms of burst release in matrix-controlled drug delivery systems. J. Control. Release 2001, 73, 121-136. [CrossRef]

29. Wada, R.; Hyon, S.; Ikada, Y. Kinetics of diffusion-mediated drug release enhanced by matrix degradation. J. Control. Release 1995, 37, 151-160. [CrossRef]

30. Zhang, L.; Li, Y.; Zhang, Y.; Zhu, C. Sustained release of isoniazid from polylactide microspheres prepared using solid/oil drug loading method for tuberculosis treatment. Sci. China Life Sci. 2016, 59, 724-731. [CrossRef]

31. Zhu, X.; Braatz, R.D. A mechanistic model for drug release in PLGA biodegradable stent coatings coupled with polymer degradation and erosion. J. Biomed. Mater. Res. Part A 2015, 103, 2269-2279. [CrossRef]

32. Mader, K.; Bittner, B.; Li, Y.; Wohlauf, W.; Kissel, T. Monitoring microviscosity and microacidity of the albumin microenvironment inside degrading microparticles from poly(lactide-co-glycolide) (PLG) or ABA-triblock polymers containing hydrophobic poly(lactide-co-glycolide) A blocks and hydrophilic poly(ethyleneoxide) B Blocks. Pharm. Res. 1998, 15, 787-793. [PubMed]

33. Mäder, K.; Crémmilleux, Y.; Domb, A.J.; Dunn, J.R.; Swartz, H.M. In Vitro/In Vivo Comparison of Drug Release and Polymer Erosion from Biodegradable P(FAD-SA) Polyanhydrides-A Noninvasive Approach by the Combined Use of Electron Paramagnetic Resonance Spectroscopy and Nuclear Magnetic Resonance Imaging. Pharm. Res. 1997, 14, 820-826. [CrossRef] [PubMed]

34. Mäder, K.; Bacic, G.; Domb, A.; Elmalak, O.; Langer, R.; Swartz, H.M. Noninvasive in Vivo Monitoring of Drug Release and Polymer Erosion from Biodegradable Polymers by EPR Spectroscopy and NMR Imaging. J. Pharm. Sci. 1997, 86, 126-134. [CrossRef] [PubMed]

35. Golubeva, E.N.; Chumakova, N.A.; Kuzin, S.V.; Grigoriev, I.A.; Kalai, T.; Korotkevich, A.A.; Bogorodsky, S.E.; Krotova, L.I.; Popov, V.K.; Lunin, V.V. Paramagnetic bioactives encapsulated in poly(D,L-lactide) microparticules: Spatial distribution and in vitro release kinetics. J. Supercrit. Fluids 2020, 158, 104748. [CrossRef]

36. Akovantseva, A.A.; Bagratashvili, V.N.; Chumakova, N.A.; Golubeva, E.N.; Gromov, O.I.; Kuzin, S.V.; Melnikov, M.Y.; Timashev, P.S. Impregnation of Polycarbonate by Paramagnetic Probe 2,2,6,6-Tetramethyl-4-Hydroxy-Piperidine-1-Oxyl (TEMPOL) in Supercritical CO 2 . Appl. Magn. Reson. 2018. [CrossRef]

37. Uddin, M.A.; Yu, H.; Wang, L.; Naveed, K.U.R.; Haq, F.; Amin, B.U.; Mehmood, S.; Nazir, A.; Xing, Y.; Shen, D. Recent progress in EPR study of spin labeled polymers and spin probed polymer systems. J. Polym. Sci. 2020, 58, 1924-1948. [CrossRef]

38. Han, X.; Ding, L.; Wang, Y.; Pan, J.; Sinka, C. A phenomenological model for the degradation of biodegradable polymers. Biomaterials 2008, 29, 3393-3401. [CrossRef]

39. Proikakis, C.S.; Mamouzelos, N.J.; Tarantili, P.A.; Andreopoulos, A.G. Swelling and hydrolytic degradation of poly(D,L-lactic acid) in aqueous solutions. Polym. Degrad. Stab. 2006, 91, 614-619. [CrossRef]

40. Khramtsov, V.V.; Weiner, L.M.; Grigoriev, I.A.; Volodarsky, L.B. Proton exchange in stable nitroxyl radicals. EPR study of the $\mathrm{pH}$ of aqueous solutions. Chem. Phys. Lett. 1982, 91, 69-72. [CrossRef]

41. Kirilyuk, I.A.; Bobko, A.A.; Khramtsov, V.V.; Grigor'ev, I.A. Nitroxides with two pK values-Useful spin probes for $\mathrm{pH}$ monitoring within a broad range. Org. Biomol. Chem. 2005, 3, 1269-1274. [CrossRef] 
42. Minaev, N.V.; Yusupov, V.I.; Bagratashvili, B.N. Laboratory Setup for the Study and Carrying Out Physical and Chemical Processes. U.S. Patent No. 147199, 20 October 2014.

43. Chumakova, N.A.; Golubeva, E.N.; Ivanova, T.A.; Vorobieva, N.N.; Timashev, P.S.; Bagratashvili, V.N. EPR Diagnostics of D,L-Polylactide Porous Matrices Formed in Supercritical $\mathrm{CO}_{2}$. Russ. J. Phys. Chem. B 2018, 12, 1255-1260. [CrossRef]

44. Eaton, G.R.; Eaton, S.S.; Barr, D.P.; Weber, R.T. Quantitative EPR; Springer: Vienna, Austria, 2010; Volume 369.

45. Chumakova, N.A.; Kuzin, S.V.; Grechishnikov, A.I. Spectral Convolution for Quantitative Analysis in EPR Spectroscopy. Appl. Magn. Reson. 2019, 50, 1125-1147. [CrossRef]

46. Duling, D.R. Simulation of Multiple Isotropic Spin-Trap EPR Spectra. J. Magn. Reson. Ser. B 1994, 104, $105-110$. [CrossRef]

47. Zaitsev, K.V.; Piskun, Y.A.; Oprunenko, Y.F.; Karlov, S.S.; Zaitseva, G.S.; Vasilenko, I.V.; Churakov, A.V.; Kostjuk, S.V. Controlled ring-opening homo- and copolymerization of $\varepsilon$-caprolactone and D,L-lactide by iminophenolate aluminum complexes: An efficient approach toward well-defined macromonomers. J. Polym. Sci. Part A Polym. Chem. 2014, 52, 1237-1250. [CrossRef]

48. Kokorin, A. (Ed.) Nitroxides-Theory, Experiment and Applications; InTech: London, UK, 2012; ISBN 978-953-51-0722-4.

49. Garlotta, D. A literature review of poly(lactic acid). J. Polym. Environ. 2001, 9, 63-84. [CrossRef]

50. Banerjee, D.; Bhat, S.N.; Bhat, S.V.; Leporini, D. ESR evidence for 2 coexisting liquid phases in deeply supercooled bulk water. Proc. Natl. Acad. Sci. USA 2009, 106, 11448-11453. [CrossRef]

51. Wang, J.; Wang, B.M.; Schwendeman, S.P. Characterization of the initial burst release of a model peptide from poly(D,L-lactide-co-glycolide) microspheres. J. Control. Release 2002, 82, 289-307. [CrossRef]

52. Messaritaki, A.; Black, S.J.; Van Der Walle, C.F.; Rigby, S.P. NMR and confocal microscopy studies of the mechanisms of burst drug release from PLGA microspheres. J. Control. Release 2005, 108, 271-281. [CrossRef]

53. Mäder, K.; Gallez, B.; Liu, K.J.; Swartz, H.M. Non-invasive in vivo characterization of release processes in biodegradable polymers by low-frequency electron paramagnetic resonance spectroscopy. Biomaterials 1996, 17, 457-461. [CrossRef]

54. Kishioka, S.Y.; Ohsaka, T.; Tokuda, K. Electrochemical studies of acid-promoted disproportionation of nitroxyl radical. Electrochim. Acta 2003, 48, 1589-1594. [CrossRef]

55. Bobko, A.A.; Kirilyuk, I.A.; Grigor'ev, I.A.; Zweier, J.L.; Khramtsov, V. V Reversible reduction of nitroxides to hydroxylamines: Roles for ascorbate and glutathione. Free Radic. Biol. Med. 2007, 42, 404-412. [CrossRef] [PubMed]

56. Jagtap, A.P.; Krstic, I.; Kunjir, N.C.; Hänsel, R.; Prisner, T.F.; Sigurdsson, S.T. Sterically shielded spin labels for in-cell EPR spectroscopy: Analysis of stability in reducing environment. Free Radic. Res. 2015, 49, 78-85. [CrossRef] [PubMed]

57. Nagura, K.; Bogdanov, A.; Chumakova, N.; Vorobiev, A.K.; Moronaga, S.; Imai, H.; Matsuda, T.; Noda, Y.; Maeda, T.; Koizumi, S.; et al. Size-tunable MRI-visible nitroxide-based magnetic mixed micelles: Preparation, stability, and theranostic application. Nanotechnology 2019, 30. [CrossRef] [PubMed]

58. Alexis, F.; Venkatraman, S.; Rath, S.K.; Gan, L.H. Some insight into hydrolytic scission mechanisms in bioerodible polyesters. J. Appl. Polym. Sci. 2006, 102, 3111-3117. [CrossRef]

59. Antheunis, H.; van der Meer, J.-C.; de Geus, M.; Kingma, W.; Koning, C.E. Improved Mathematical Model for the Hydrolytic Degradation of Aliphatic Polyesters. Macromolecules 2009, 42, 2462-2471. [CrossRef]

60. Xu, Y.; Kim, C.S.; Saylor, D.M.; Koo, D. Polymer degradation and drug delivery in PLGA-based drug-polymer applications: A review of experiments and theories. J. Biomed. Mater. Res. Part B Appl. Biomater. 2017, 105, 1692-1716. [CrossRef] [PubMed]

61. Fredenberg, S.; Wahlgren, M.; Reslow, M.; Axelsson, A. The mechanisms of drug release in poly(lactic-co-glycolic acid)-based drug delivery systems-A review. Int. J. Pharm. 2011, 415, 34-52. [CrossRef]

62. Huang, J.; Mazzara, J.M.; Schwendeman, S.P.; Thouless, M.D. Self-healing of pores in PLGAs. J. Control. Release 2015, 206, 20-29. [CrossRef]

63. Reinhold, S.E.; Desai, K.G.H.; Zhang, L.; Olsen, K.F.; Schwendeman, S.P. Self-healing microencapsulation of biomacromolecules without organic solvents. Angew. Chem. Int. Ed. 2012, 51, 10800-10803. [CrossRef] 
64. Antheunis, H.; van der Meer, J.-C.; de Geus, M.; Heise, A.; Koning, C.E. Autocatalytic Equation Describing the Change in Molecular Weight during Hydrolytic Degradation of Aliphatic Polyesters. Biomacromolecules 2010, 11, 1118-1124. [CrossRef]

65. Borovikov, P.I.; Sviridov, A.P.; Antonov, E.N.; Dunaev, A.G.; Krotova, L.I.; Fatkhudinov, T.K.; Popov, V.K. Model of aliphatic polyesters hydrolysis comprising water and oligomers diffusion. Polym. Degrad. Stab. 2019, 159, 70-78. [CrossRef]

66. Siparsky, G.L.; Voorhees, K.J.; Dorgan, J.R.; Schilling, K. Water Transport in Polylactic Acid (PLA), PLA/Polycaprolactone Copolymers, and PLA/Polyethylene Glycol Blends. J. Environ. Polym. Degrad. 1997, 5, 125-136.

67. Sevim, K.; Pan, J. A model for hydrolytic degradation and erosion of biodegradable polymers. Acta Biomater. 2018, 66, 192-199. [CrossRef] [PubMed]

68. Li, S.M.; Garreau, H.; Vert, M. Structure-property relationships in the case of the degradation of massive aliphatic poly-( $\alpha$-hydroxy acids) in aqueous media-Part 1: Poly(dl-lactic acid). J. Mater. Sci. Mater. Med. 1990, 1, 123-130. [CrossRef]

69. Jonnalagadda, S.; Robinson, D.H. Effect of thickness and PEG addition on the hydrolytic degradation of PLLA. J. Biomater. Sci. Polym. Ed. 2004, 15, 1317-1326. [CrossRef]

70. Grizzi, I.; Garreau, H.; Li, S.; Vert, M. Hydrolytic degradation of devices based on poly(dl-lactic acid) size-dependence. Biomaterials 1995, 16, 305-311. [CrossRef]

Publisher's Note: MDPI stays neutral with regard to jurisdictional claims in published maps and institutional affiliations. 